\title{
NUMERICAL MINIMIZATION OF EIGENMODES OF A MEMBRANE WITH RESPECT TO THE DOMAIN
}

\author{
Édouard OUdeT ${ }^{1}$
}

\begin{abstract}
In this paper we introduce a numerical approach adapted to the minimization of the eigenmodes of a membrane with respect to the domain. This method is based on the combination of the Level Set method of S. Osher and J.A. Sethian with the relaxed approach. This algorithm enables both changing the topology and working on a fixed regular grid.
\end{abstract}

Mathematics Subject Classification. 1991.

Received January 15, 2003. Revised October 10, 2003.

\section{Statement AND historical SUMmary}

Let $\Omega$ be a bounded open set of $\mathbb{R}^{N}$ and $H_{0}^{1}(\Omega)$ the Sobolev space defined as the closure of $C^{\infty}$ functions with compact support in $\Omega$ with respect to the norm

$$
\|u\|_{H^{1}}:=\left(\int_{\Omega} u(x)^{2} \mathrm{~d} x+\int_{\Omega}|\nabla u(x)|^{2} \mathrm{~d} x\right)^{1 / 2} .
$$

The Laplace-Dirichlet operator on $\Omega$ being a self-adjoint operator with compact inverse, there is a sequence of positive eigenvalues which tends to $+\infty$ and an associated sequence of eigenvectors that will be labelled $0<\lambda_{1}(\Omega) \leq \lambda_{2}(\Omega) \leq \lambda_{3}(\Omega) \leq \ldots$ and $u_{1}, u_{2}, u_{3}, \ldots$ Thus, those sequences solve the problems

$$
\begin{cases}-\Delta u_{k}=\lambda_{k}(\Omega) u_{k} & \text { in } \Omega \\ u_{k}=0 & \text { on } \partial \Omega\end{cases}
$$

In any case, the eigenfunctions will be supposed to satisfy the condition

$$
\int_{\Omega} u_{k}(x)^{2} \mathrm{~d} x=1
$$

The eigenfunctions constitute an Hilbert base of $L^{2}(\Omega)$.As a consequence of the hypo-analyticity of the laplacian

Keywords and phrases. Shape optimization, eigenvalue, level set, relaxation.

1 Institut de Recherche Mathématique Avancée, Université Louis Pasteur, 67084 Strasbourg Cedex, France;

e-mail: Edouart.Oudet@univ-savoie.fr

(c) EDP Sciences, SMAI 2004 
they are analytic in $\Omega$. Moreover, we shall use the following variational formulation:

$$
\lambda_{1}(\Omega)=\inf _{v \in H_{0}^{1}(\Omega), v \neq 0} \frac{\int_{\Omega}|\nabla v(x)|^{2} \mathrm{~d} x}{\int_{\Omega} v(x)^{2} \mathrm{~d} x} .
$$

This infimum is reached when $v=u_{1}$, the first eigenfunction of the Laplace-Dirichlet operator.

The investigations of the relations between the eigenmodes of the domain $\Omega$, and specifically the isoperimetric inequalities where they are involved, are an important issue both in the field of partial differential equations and in differential geometry. In the beginning of the century, the first result obtained in this topic was the proof of the Rayleigh conjecture by Faber [7] and Krahn [14]. They proved that the ball minimizes the first eigenvalue of the Laplace-Dirichlet operator under a volume constraint. The domain which minimizes $\lambda_{2}$ always with a volume constraint is the union of two identical balls. This result was often attributed to Szegö as observed by Pòlya in [20], but it seems that this result was already published in a paper of Krahn (see [15]). For $k \geq 3$ fixed, the question to identify the open set of the plane which minimizes $\lambda_{k}$ amongst the sets of given area remains open today (see [10] for a survey on this subject).

In 1973, Troesch proposes in [23] some numerical values of resonant frequencies for several types of convex membranes of $\mathbb{R}^{2}$. The following observations stand out from those experiments:

- the convex open set which minimizes the second eigenvalue of the Laplace-Dirichlet operator under convexity and volume constraints displays two flat sections on its boundary;

- the stadium (see Fig. 1), i.e. the convex hull of two tangent identical disks, looks very close to the optimal set.

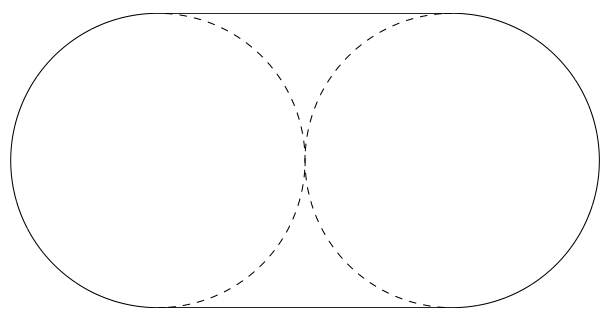

Figure 1. One stadium.

In fact, as reported in [11] and [12], the stadium does not minimizes $\lambda_{2}$ under convexity and volume constraints. The aim of this work is to present a new technique enabling to approximate numerically the solutions of such optimizations problems. More precisely, we shall be interested in the two following problems:

$$
\begin{gathered}
\min \left\{\lambda_{2}(\Omega), \Omega \subset \mathbb{R}^{2}, \Omega \text { convex, }|\Omega|=1\right\} \\
\min \left\{\lambda_{k}(\Omega), \Omega \subset \mathbb{R}^{2},|\Omega|=1\right\} \text { for } k \geq 3 .
\end{gathered}
$$

The method we are presenting combines two approaches that were generated in the last twenty years, respectively the homogenization method and the level set method. We shall start with a short description of the three main numerical methods in shape optimization, namely the boundary variation, the homogenization and the level set methods. For each of them we shall underline the drawbacks when applying those techniques to minimize the eigenmodes of the Laplace operator. In consequence we shall develop a new process.

In conclusion we shall report numerical results. On one side we improve the values published in [24] and on the other side we propose a geometrical description of the ten first optimal sets.

\section{Boundary VARIATION, DERIVATIVE With RESPECT TO THE DOMAIN}

The boundary variation method is the first we used for our study of the eigenvalues. This allowed to obtain satisfying results when minimizing $\lambda_{2}$ under volume and convexity constraints. This approach turns out to be 
ineffective to investigate directly the minimization under volume constraint only, but unlike the technique that will be introduced later, it does not require to modify the cost function.

\subsection{General presentation}

Many optimization methods are founded on the use of the so-called first order optimality conditions (as the gradient and quasi-Newton methods). These techniques are descent methods which we try to make converge to a local minimum of the cost function. The question which arises in shape optimization is the deficiency of a natural derivative in a space of shapes. To by-pass this difficulty we introduce for each vector field $V$ (so-called deformation field) of $\mathbb{R}^{N}$, the derivative (if it does exist) of $J$ in the direction $V$ by

$$
d(J(\Omega))(\Omega, V)=\lim _{t \rightarrow 0} \frac{J\left(\Omega_{t}\right)-J\left(\Omega_{0}\right)}{t},
$$

where

$$
\Omega_{t}=\{(I d+t V)(x), x \in \Omega\} .
$$

A function $J$ which displays an extremum in $\Omega$ satisfies the relation

$$
d(J(\Omega))(\Omega, V)=0
$$

for all vector fields $V$.

Remark 1. Under some regularity assumptions it can be proved (see [22]) that

$$
\exists h_{\Omega} \in \mathcal{D}^{\prime}(\partial \Omega) \text { such that } \forall V \in \mathcal{D}\left(\mathbb{R}^{N}\right)^{N}, d(J(\Omega))(\Omega, V)=<h_{\Omega}, V \cdot n>_{\mathcal{D}^{\prime}(\partial \Omega) \times \mathcal{D}(\Omega)},
$$

where $n$ is the normal vector field of $\partial \Omega$. This result expresses that the boundary derivative only takes into account the normal component of the deformation field.

We shall now specify the relation (6) in the frame of eigenvalue problems. More precisely let us report the following Hadamard's theorem:

Theorem 2 (Hadamard). Let $\Omega$ be an open set of class $C^{2}$ and $\lambda_{k}(\Omega)$ be its kth eigenvalue of the LaplaceDirichlet operator. We assume that $\lambda_{k}(\Omega)$ is a simple eigenvalue. Then the function $t \mapsto \lambda_{k}\left(\Omega_{t}\right)$ has a derivative at $t=0$, which is given by

$$
d\left(\lambda_{k}(\Omega)\right)(\Omega, V)=-\int_{\partial \Omega}\left(\frac{\partial u_{k}}{\partial n}\right)^{2} V \cdot n \mathrm{~d} \sigma
$$

where $u_{k}$ is the $k^{\text {th }}$ eigenfunction associated to $\lambda_{k}(\Omega)$, normalized by $\int_{\Omega} u_{k}^{2}(x) \mathrm{d} x=1$.

A complete proof of this result is reported in [22] or in [13]. Here we will only present a formal proof of the relation (7). Let $u_{k}(t, x)$ and $\lambda_{k}\left(\Omega_{t}\right)$ be the $k$ th eigenfunction and eigenvalue of the open set $\Omega_{t}$. We suppose that the derivative with respect to the real parameter $t$ exists. Deriving formally at $t=0$ the relation

$$
-\triangle u_{k}=\lambda_{k}\left(\Omega_{t}\right) u_{k}
$$

we obtain

And the boundary condition

$$
-\triangle u_{k}^{\prime}=\lambda_{k}(\Omega) u_{k}^{\prime}+\lambda_{k}^{\prime}(\Omega) u_{k} \text { in } \Omega
$$

becomes

$$
u_{k}(t,(I d+t V)(x))=0 \text { for all } x \in \partial \Omega
$$

$$
u_{k}^{\prime}+\sum_{i=1}^{N} \frac{\partial u_{k}}{\partial x_{i}} \frac{\mathrm{d}(I d+t V)_{i}}{\mathrm{~d} t}=u_{k}^{\prime}+\sum_{i=1}^{N} \frac{\partial u_{k}}{\partial x_{i}} V_{i}=0 .
$$


Then

$$
u_{k}^{\prime}=-\frac{\partial u_{k}}{\partial n} V \cdot n
$$

since $\nabla u_{k}=\frac{\partial u_{k}}{\partial n} \cdot n$ ( $u_{k}$ is constant on the boundary). Multiplying (9) by $u_{k}$ and integrating on $\Omega$ we obtain

$$
-\int_{\Omega} u_{k} \triangle u_{k}^{\prime} \mathrm{d} x=\lambda_{k}(\Omega) \int_{\Omega} u_{k} u_{k}^{\prime} \mathrm{d} x+\lambda_{k}^{\prime}(\Omega)
$$

Hence by Green's formula,

$$
-\int_{\partial \Omega} \frac{\partial u_{k}^{\prime}}{\partial n} u_{k} \mathrm{~d} \sigma+\int_{\partial \Omega} \frac{\partial u_{k}}{\partial n} u_{k}^{\prime} \mathrm{d} \sigma-\int_{\Omega} u_{k}^{\prime} \triangle u_{k} \mathrm{~d} x=\lambda_{k}(\Omega) \int_{\Omega} u_{k} u_{k}^{\prime} \mathrm{d} x+\lambda_{k}^{\prime}(\Omega) .
$$

Moreover multiplying (8) at $t=0$ by $u_{k}^{\prime}$ and integrating on $\Omega$, we get the new relation

$$
-\int_{\Omega} u_{k}^{\prime} \triangle u_{k} \mathrm{~d} x=\lambda_{k}(\Omega) \int_{\Omega} u_{k} u_{k}^{\prime} \mathrm{d} x
$$

From (10)-(12) and the fact that $u_{k}$ equals zero on the boundary, we conclude that

$$
d(J(\Omega))(\Omega, V)=\lambda_{k}^{\prime}(\Omega)=-\int_{\partial \Omega}\left(\frac{\partial u_{k}}{\partial n}\right)^{2} V \cdot n \mathrm{~d} \sigma .
$$

Remark 3. According to the formula $\lambda_{k}(l \Omega)=\frac{\lambda_{k}(\Omega)}{l^{2}}$ (for $l>0$ ), we can neglect the volume constraint in the problems (4) and (5). We have:

$$
\min _{|\Omega|=1, \Omega \text { open }} \lambda_{k}(\Omega)=\min _{\Omega \text { open }}|\Omega| \lambda_{k}(\Omega)
$$

To estimate the derivative of $|\Omega| \lambda_{k}(\Omega)$, we have to compute the derivative of the volume with respect to a boundary variation. Such a result is easily obtained (see as an example [13]) going back to a fixed domain by Green's formula. Then

$$
d(|\Omega|)(\Omega, V)=\int_{\partial \Omega} V \cdot n \mathrm{~d} \sigma
$$

Thanks to this derivative we are able to describe the different steps of a boundary optimization algorithm designed to minimize the $k$ th eigenvalue under volume constraint:

- Polygonal discretization of the boundary: let $\Omega_{0}$ be a given initial shape; $m$ control points on $\partial \Omega_{0}$ are selected and referred to as $P_{i} i=1, \ldots, m$;

- Computation of the shape derivative: let $n\left(P_{i}\right)$ be the exterior normal vector at the point $P_{i}$. Thanks to $(7)$ and (13), for each $i=1, \ldots, m$, we compute the derivative

$$
d_{i}=\lim _{t \rightarrow 0} \frac{J\left(\Omega_{t}^{i}\right)-J\left(\Omega_{0}\right)}{t}
$$

of the function of one variable $J\left(\Omega_{t}^{i}\right)$, where

$$
\left\{\begin{array}{l}
J(\Omega)=|\Omega| \lambda_{k}(\Omega) \\
\Omega_{t}^{i}=\left\{\left(I d+t V_{i}\right)(x), x \in \Omega\right\}
\end{array}\right.
$$

for $V_{i}=v_{i}(x) n(x)$ where $v_{i}$ is a smooth real-valued function equal to 1 at $P_{i}$ and zero on all other control points. Therefore we make use of the approach reported for example in [19] about the problem of electromagnetic shaping;

- Exit criterion: if the norm of the vector $\left(d_{i}\right)_{i=1, \ldots, m}$ is small enough we stop the algorithm; 
- Boundary variation: each control point is moved along the exterior normal vector according to the opposite of the derivative $d_{i}$. Precisely, $P_{i}$ is translated from a vector $-\alpha d_{i} n\left(P_{i}\right)$ for an adaptive choice of $\alpha>0$. In this context the quasi-Newton method can also be carried out (see [19]);

- Evaluation of the cost function: after having verified that the new control points $\left(P_{i}\right)_{i=1, \ldots, m}$ still generates a non crossing polygon, the value of the cost function for this new shape is evaluated. Then the descent step $\alpha$ is adjusted exactly the same way as in the finite dimension case for a classical gradient algorithm. An iteration takes end by going back to the step of derivative computation.

This method has been carried out in numerous situations different from the minimization of eigenvalues. However, it displays three major drawbacks that are respectively:

- no change of the topology;

- the risk to get a local minimum;

- a major computational cost due to the remeshing process for each iteration.

However this method was shown to be efficient for the investigation of the second eigenvalue under convexity and volume constraints.

\subsection{Application to the problem of the stadium}

Let's go back to the problem (4). In [12] we could prove that the shape optimization problem

$$
\min _{\Omega \text { convex, }|\Omega|=1} \lambda_{2}(\Omega)=\min _{\Omega} \lambda_{\text {convex }}(\Omega)|\Omega|
$$

is well-posed and that the stadium does not realize this minimum. Moreover we have shown that the optimal set has exactly two parallel segments on its boundary.

Here we wish to report a computational description of the optimal shape using the boundary variation method. The only difficulty we still have to deal with is taking into account the convexity constraint. Therefore the boundary variation algorithm is applied to the penalized function

$$
\bar{J}(\Omega):=\lambda_{2}(\Omega)|\Omega|+p(|C o(\Omega)|-|\Omega|)^{2}
$$

where $C o(\Omega)$ is the convex hull of $\Omega$ and $p$ is a positive penalization number. In Figures 2 and 3 we present the results obtained with two distinct initial shapes. Figure 2 illustrates the convex hulls of the shapes generated by the boundary variation method. This post-processing has been involved since the penalization method does not generate exact convex shapes. We observe the close similarity between a stadium and an optimal shape both from the geometrical viewpoint and from their eigenvalues (see Tab. 1 where $j_{0} \simeq 2.4048256$ and $j_{1} \simeq 3.8317060$ are respectively the first zeros of the Bessel functions $J_{0}$ and $J_{1}$ ).

\subsection{Remarks and precisions}

In the previous computations, the boundary was discretized by 40 control points. We used classical finite element method to approximate the eigenvalues of the laplacian. Since we need a good approximation of the gradient of the eigenfunction on the boundary, we used a $P 2$ finite element method with about 4000 triangles. In the cases of the disc or of the square as initial shapes, convergence takes place in less than 40 iterations.

Alternatively, we attempted to change the penalization approach for a projection method. At each iteration the new open set was replaced by its convex hull. Definitely this process was rather unefficient. The main reason is the following: taking the convex hull introduces segments on the boundary. Now, in many cases, the boundary variation method would like to push the nodes on the segment inside: then taking again the convex hull will restore the initial domain. At this point the method is stationary. 


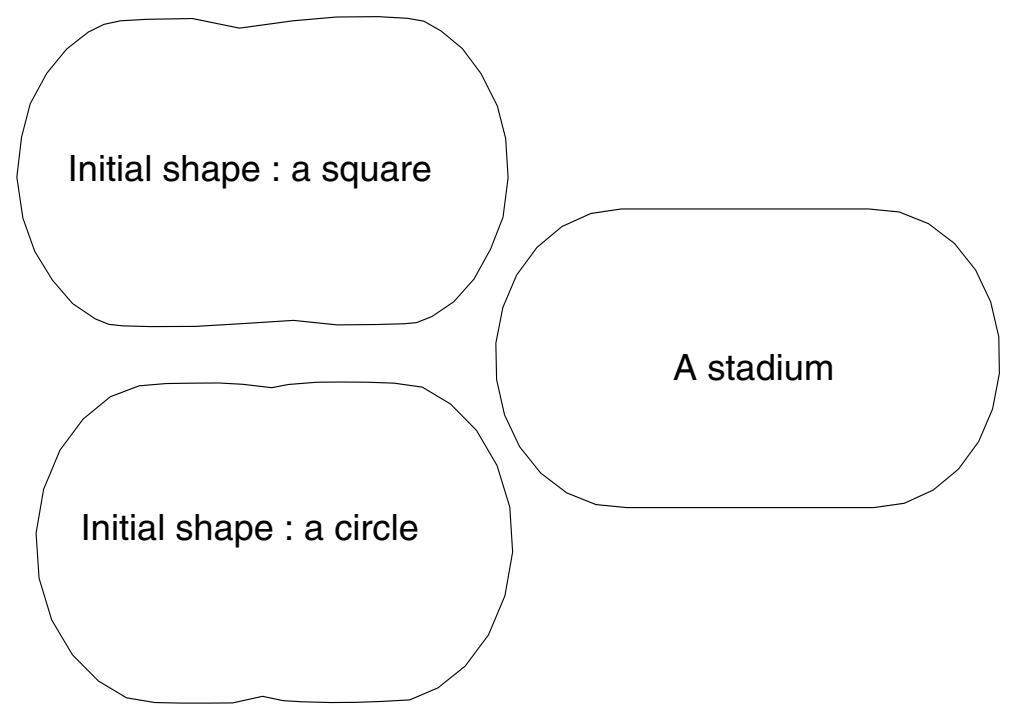

FIGURE 2. Optimization obtained for several initial shapes.

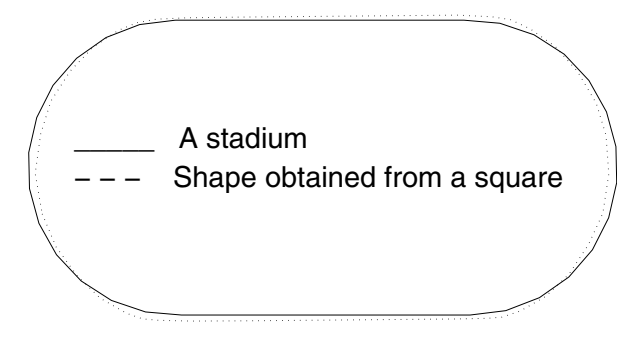

Figure 3. A test of superposition.

TABLE 1. Different values of $\lambda_{2}$.

\begin{tabular}{l|c|c}
\hline Domain & Exact value & Computed value \\
\hline Square & $5 \pi^{2}$ & 49.348 \\
Ball & $j_{1}^{2} \pi$ & 46.124 \\
Best rectangle & $4 \pi^{2}$ & 39.478 \\
Best ellipse & & 39.317 \\
The stadium & & 38.001 \\
The best convex domain & & 37.980 \\
Two balls $^{2}$ & & 36.336 \\
\hline
\end{tabular}

${ }^{1}$ That we have obtained with our algorithm.

${ }^{2}$ Best domain (without constraint).

\section{THE RELAXED APPROACH}

\subsection{Introduction}

We are now presenting a relaxed method by homogenization which was very successful during the last years both for obtaining existence results in shape optimization and developing numerical algorithms. 
Numerous shape optimization problems have no solution in the natural space of investigation (typically the open subsets of a compact domain). As well as weak solutions have been introduced in the field of partial differential equations, we are led to enlarge the space of shapes in order to obtain the existence of an optimal generalized shape. This process was applied mainly in elastic structures (see $[1,3,4]$ ), where the expected domain is deduced from the homogenized solution after a screening technique.

\subsection{The relaxed approach for eigenmodes}

Let $D$ be a bounded regular open set of $\mathbb{R}^{N}$ and $\Omega$ an open set of $D$. For $k \in \mathbb{N}^{*}$, we consider $u_{\Omega} \in H_{0}^{1}(\Omega)$ as the $k$ th eigenvalue of the laplacian. Therefore $u_{\Omega}$ is a solution of

$$
\left\{\begin{array}{l}
-\Delta u_{\Omega}=\lambda_{k}(\Omega) u_{\Omega} \text { in } \Omega \\
u_{\Omega}=0 \text { on } \partial \Omega
\end{array}\right.
$$

We are interested in the shape optimization problem (5). Apart from $k=1$ and 2 , it is not yet established whether such a problem has an open set solution. An important result in this field was obtained by Buttazzo and Dal Maso (see [5]) who proved that a quasi open set solution of (5) does exist.

Hereunder we will introduce a relaxed approach adapted to the problem (5). For each $\mu$ a non-negative Borel measure on $D$ we consider the new eigenvalue problem as follows:

$$
\left\{\begin{array}{l}
-\Delta u+\mu u=\lambda(\mu) u \text { in } D \\
u=0 \text { on } \partial D
\end{array}\right.
$$

By analogy with the classical weak solution of partial differential equations we shall define a solution $(\lambda, u)$ of (14) as a pair which satisfies

$$
\left\{\begin{array}{l}
\lambda \in \mathbb{R}, u \in V_{\mu}^{0}, \\
\int_{D} \nabla u(x) \cdot \nabla v(x) \mathrm{d} x+\int_{D} u(x) v(x) \mathrm{d} \mu=\lambda(\mu) \int_{D} u(x) v(x) \mathrm{d} x, \forall v \in V_{\mu}^{0},
\end{array}\right.
$$

where $V_{\mu}^{0}=H_{0}^{1}(D) \cap L_{\mu}^{2}(D)$ and $L_{\mu}^{2}(D)$ is the set of measurable functions whose square value admits a finite integral with respect to $\mu$. It is easily shown that for each $\mu \in \mathcal{M}_{0}(D)$ (the set of non-negative Borel measure on $D$ absolutely continuous with respect to capacity) there are sequences of eigenvectors and eigenvalues satisfying (15). Let us now develop how to associate for each open set in $D$ an element of $\mathcal{M}_{0}(D)$. Therefore we consider the capacity measure on $D$ defined for all $E \in \mathcal{P}(D)$ (the subsets of $D$ ) by:

$$
\operatorname{cap}_{D}(E)=\inf \left\{\int_{D}|\nabla u|^{2} \mathrm{~d} x: u \in C_{0}^{\infty}(D), u \geq 1 \text { in a neighborhood of } E\right\} .
$$

For each $\Omega \subset D$, we introduce $\mu_{\Omega} \in \mathcal{M}_{0}(D)$ by

$$
\mu_{\Omega}(B)=\left\{\begin{array}{l}
0 \text { if } \operatorname{cap}_{D}(B \backslash \Omega)=0 \\
+\infty \text { if } \operatorname{cap}_{D}(B \backslash \Omega)>0
\end{array}\right.
$$

for each borelian set $B$ of $D$. So a natural injection of the open sets of $D$ into $\mathcal{M}_{0}(D)$ is built. We still have to define the volume of an element of $\mathcal{M}_{0}(D)$. Let us define for each $\mu \in \mathcal{M}_{0}(D)$

$$
|\mu|=\left|\left\{x \in D, w_{\mu}(x)>0\right\}\right|
$$

where $w_{\mu}$ is the weak solution of

$$
\left\{\begin{array}{l}
-\Delta w_{\mu}+\mu w_{\mu}=1 \text { in } D \\
w_{\mu} \in H_{0}^{1}(D)
\end{array}\right.
$$


Remark 4. The expected equality $\left|\mu_{\Omega}\right|=|\Omega|$ is deduced from the maximum principle and the definition (17). Indeed the function $w_{\mu_{\Omega}}$ is formally zero outside $\Omega$ and sub-harmonic inside.

The following theorem can thus be established (see [8] or [13] for the proof and also for a precise definition of the $\gamma$-convergence):

Theorem 5 (Dal Maso-Mosco). The set $\left\{\mu \in \mathcal{M}_{0}(D),|\mu| \leq 1\right\}$ is the completion with respect to the $\gamma$ convergence of the family of open sets of $D$ whose volume are less than 1 . Moreover, $\mathcal{M}_{0}(D)$ is compact for this topology.

Having proved the continuity of the eigenvalues with respect to the $\gamma$-convergence and the monotonicity of $\lambda_{k}(\Omega)$ with respect to set inclusion, it can be shown that the following problem

$$
\inf _{\mu \in \mathcal{M}_{0}(D),|\mu| \leq 1} \lambda_{k}(\mu)
$$

does admit a solution. In addition, this solution is a classical domain and not only a measure in $\mathcal{M}_{0}(D)$.

\subsection{Numerical approximation}

In this section we shall show how the relaxed formulation (14) is well suited for finding an optimal shape. This method however exhibits an important practical difficulty: in such a context the volume of a measure appears to be non differentiable under its variation. A first application of this method for the minimization of eigenvalues was reported in [8]. In this paper the author by-passes this difficulty by a smoothing process. Unfortunately such a method requires the introduction in the algorithm of parameters whose adjustment proved to be delicate. For this reason we did not apply this method directly and preferred a mixed method that will be described in the following paragraph.

Let $\left(T_{h}\right)_{h=1, \ldots, l}$ be a triangulation of $D$. We aim to approximate the measure solution of (19) by a sequence of absolute continuous measures with respect to the Lebesgue measure whose densities are constant on each $T_{h}$. Having applied the algorithm we expect a measure of type (17), i.e. a zero or "infinity" value measure on almost each triangle (at least on each triangle which are not on the interface region). Let $\left(m_{h}\right)_{h=1, \ldots, l} \in \mathbb{R}_{+}^{l}$ be the measure whose density is given by

$$
\sum_{h=1}^{l} m_{h} \chi_{T_{h}}
$$

where $\chi_{T_{h}}$ is the characteristic function of the triangle $T_{h}$. Let us recall from [8] the following proposition (such a formula can be obtained exactly as in Sect. 2).

Proposition 1. Let $\mu \in \mathcal{M}_{0}(D)$ with density $\left(m_{h}\right)_{h=1, \ldots, l}$ and $k \in \mathbb{N}^{*}$. Let $\lambda_{k}(\mu)$ i.e. the kth eigenvalue of the operator $-\Delta+\mu I$, be a simple eigenvalue and $u_{k}$ its eigenfunction. Then

$$
\frac{\partial \lambda_{k}}{\partial m_{h}}=\int_{T_{h}}\left(u_{k}\right)^{2} \mathrm{~d} x
$$

Having computed this derivative, the aim is to apply a classical gradient algorithm to the sequence $\left(m_{h}\right)_{h=1, \ldots, l}$ (exactly the same way we optimized the positions of control points here above). As it has been already mentioned, the volume of a measure $\mu$ is not differentiable with respect to $m_{h}$. This problem is removed when the level set method is carried out as shown in the next section. Moreover, this level set method allows us to consider measures which take only the values 0 and "infinity" (i.e. a large value $M$, see below). 


\section{THE LEVEL SET METHOD AND THE RELAXED FORMULATION}

\subsection{Introduction}

The method that will be reported is adapted from a paper of Osher and Santosa on problems of not degenerated densities (see [16]). A level set method exhibits several advantages. In particular it does not imply any topological restriction and enables working on a fixed regular mesh. Before detailing the different steps, let us recall the principle of a level set method. Let $\Omega \subset D$ be an initial shape. The first step will be to parametrize $\Omega$ by a function $\Phi$, the so-called level set function, that must satisfy

$$
\left\{\begin{array}{l}
\Phi(x)<0 \text { if } x \in \Omega \\
\Phi(x)>0 \text { if } x \in D \backslash \bar{\Omega} \\
\Phi(x)=0 \text { if } x \in \partial \Omega
\end{array}\right.
$$

Next, as suggested in [17], such a function will be initialized with the signed-distance which is given by

$$
\left\{\begin{array}{l}
\Phi(x)=-\operatorname{dist}(x, \partial \Omega) \text { if } x \in \Omega, \\
\Phi(x)=\operatorname{dist}(x, \partial \Omega) \text { if } x \in D \backslash \Omega .
\end{array}\right.
$$

Let us observe that the constructed distance is generally not easily computed and often requires a specific attention (see [21]). As far as we are concerned, the initial shapes have been estimated thanks to genetic techniques (see Sect. 4.4). We choose an approximate signed-distance function which is constant on each triangle of the mesh. Its value in the triangle $T$ is computed by evaluating the distance between the center of mass of $T$ and the center of mass of the closest triangle lying on the boundary of the initial shape.

One $\Phi$ is defined, let its the level set 0 (i.e. $\partial \Omega$ ) fluctuate with time under the vector field $v n$ (where $v$ is a realvalued function). In other words, if $x(t)$ describes the evolution of a point on $\partial \Omega$ under such a transformation, it has to verify

$$
\Phi(t, x(t))=0 \text { for all } t
$$

Differentiating this expression, we obtain

$$
\frac{\partial \Phi}{\partial t}(t, x(t))+\frac{\mathrm{d} x}{\mathrm{~d} t}(t) \cdot \nabla_{x} \Phi\left(t,(t, x(t))=\frac{\partial \Phi}{\partial t}(t, x(t))+v(x(t)) n(x(t)) \cdot \nabla_{x} \Phi(t, x(t))=0\right.
$$

Now the normal to a level set in a non stationary point is given by

$$
n(x(t))=\frac{\nabla_{x} \Phi}{\left|\nabla_{x} \Phi\right|}(t, x(t)) .
$$

Hence according to (20),

$$
\frac{\partial \Phi}{\partial t}(t, x(t))+v(x(t))\left|\nabla_{x} \Phi\right|(t, x(t))=0 .
$$

In order to compute the evolution of $\Phi$, we thus have to solve a Hamilton-Jacobi equation. It has to be mentioned that the computation we have presented only concerns the level set 0 . But since the vector field vn has a natural extension on a $D$, we solve the equation $(21)$ in the whole set $D$.

A major difficulty, that we did not yet mention and which will be treated in the next paragraph, is the computation of a good velocity field $v n$ for the shape optimization problem under investigation. For this purpose, a very natural approach has been introduced in [2], which consists in choosing the vector field as the field obtained by boundary variation.

Before going into details, let us summarize the different steps of the level set optimization:

1. initialization of $\Phi$ by the signed distance;

2. computation of the velocity field and checking of an exit criterion;

3. propagation of the level set solving the Hamilton-Jacobi equation (21); 
4. evaluation of the cost function;

5. redefinition of $\Phi$ and adjustment of the time step;

6. eventually, reinitialization of $\Phi$ with the signed distance. Back to step 2.

\subsection{Computation of the derivative}

We here report the computation of the variation of the eigenvalue with respect to $\Phi$. Here again, we only justify formally the derivative. We refer to [13] and [22] for a rigorous proof.

Let $\mu$ be a density on $D$ having only 0 or $M$ values. We notice that for $M$ large enough, the value of $\lambda_{k}(\mu)$ is closed of $\lambda_{k}(\Omega)$ where $\Omega=\{x \in D: \mu(x)=0\}$. We have to estimate the derivative of $\lambda_{k}(\mu)$ with respect to a variation of its level set subjected to a normal vector field $v n$. Let $k \in \mathbb{N}^{*}$ and $u_{\mu}$ the solution of

$$
\left\{\begin{array}{l}
-\Delta u_{\mu}+\mu u_{\mu}=\lambda_{k}(\mu) u_{\mu} \text { in } D \\
u_{\mu}=0 \text { on } \partial D
\end{array}\right.
$$

according to the preceding paragraph. Let $u_{\mu, t}$ verify

$$
\left\{\begin{array}{l}
-\Delta u_{\mu, t}+\mu_{t} u_{\mu, t}=\lambda_{k}\left(\mu_{t}\right) u_{\mu, t} \text { in } D \\
u_{\mu, t}=0 \text { on } \partial D
\end{array}\right.
$$

where, for each $x \in D$,

$$
\mu_{t}(x)=\left\{\begin{array}{l}
0 \text { for } x \in\{(I d+t v n)(y): \mu(y)=0\} \\
M \text { otherwise. }
\end{array}\right.
$$

The derivability of $\lambda_{k}\left(\mu_{t}\right)$ and $u_{\mu, t}$ with respect to $v n$ can be proved as in [22] and [13]. Once this derivability admitted, let us establish the formula of the derivative.

After multiplying (23) by a test function $w \in C_{0}^{\infty}(D)$ and by integrating it using Green's formula we obtain the weak formulation

$$
\int_{D} \nabla u_{\mu, t} \cdot \nabla w \mathrm{~d} x+\int_{D} u_{\mu, t} w \mathrm{~d} \mu_{t}=\lambda_{k}\left(\mu_{t}\right) \int_{D} u_{\mu, t} w \mathrm{~d} x
$$

for each $w \in C_{0}^{\infty}(D)$. Hence

$$
\int_{D} \nabla u_{\mu, t} \cdot \nabla w \mathrm{~d} x+M \int_{\omega_{t}} u_{\mu, t} w \mathrm{~d} x=\lambda_{k}\left(\mu_{t}\right) \int_{D} u_{\mu, t} w \mathrm{~d} x
$$

where

$$
\omega_{t}=\left\{x \in D: \mu_{t}(x)=M\right\} .
$$

Deriving this latter identity with respect to $t$ and using classical identities, we have

$$
\int_{D} \nabla u_{\mu}^{\prime} \cdot \nabla w \mathrm{~d} x+M\left(\int_{\omega} u_{\mu}^{\prime} w \mathrm{~d} x-\int_{\partial \omega} u_{\mu} w v \mathrm{~d} \sigma\right)=\lambda_{k}^{\prime}(\mu) \int_{D} u_{\mu} w \mathrm{~d} x+\lambda_{k}(\mu) \int_{D} u_{\mu}^{\prime} w \mathrm{~d} x,
$$

where $\omega$ stands for $D \backslash \Omega$ (i.e. $\omega_{t}$ fot $t=0$ ). In order to eliminate $u_{\mu}^{\prime}$ we evaluate (24) when $t=0$ and $w=u_{\mu}^{\prime}$ and we deduce

$$
\int_{D} \nabla u_{\mu} \cdot \nabla u_{\mu}^{\prime} \mathrm{d} x+\int_{D} u_{\mu} u_{\mu}^{\prime} \mathrm{d} \mu=\lambda_{k}(\mu) \int_{D} u_{\mu} u_{\mu}^{\prime} \mathrm{d} x .
$$

Combining (25) with $w=u_{\mu}$ and (26), we get

$$
\lambda_{k}^{\prime}(\mu) \int_{D} u_{\mu}^{2} \mathrm{~d} x=-M \int_{\partial \omega} u_{\mu}^{2} v \mathrm{~d} \sigma
$$


SO

$$
\lambda_{k}^{\prime}(\mu)=-\frac{M \int_{\partial \omega} u_{\mu}^{2} v \mathrm{~d} \sigma}{\int_{D} u_{\mu}^{2} \mathrm{~d} x}=-M \int_{\partial \omega} u_{\mu}^{2} v \mathrm{~d} \sigma
$$

for a normalized function $u_{\mu}$.

Exactly in the same way as under boundary variation, the derivative of the measure's volume can be evaluated as:

$$
|\mu|^{\prime}=\int_{\partial \omega} v(x) \mathrm{d} \sigma .
$$

\subsection{Computing a numerical solution of Hamilton-Jacobi equation}

Now, the problem is to determine amongst the weak solutions the one that corresponds to the physical state under investigation. Introducing the concept of viscosity solution, in 1983 Crandall and Lions (see [6]), brought a satisfying answer to the global existence problem for Hamilton-Jacobi equations. We shall not go into technical details for the definition of such a weak solution (we refer to [6] or [21]).

Our description will be limited to an algorithm reported in [17] designed to approach the weak viscosity solution of our problem.

Let us consider the first order Cauchy's system:

$$
\left\{\begin{array}{l}
\frac{\partial \Phi}{\partial t}(t, x)-F(x)|\nabla \Phi(t, x)|=0 \text { in } \mathbb{R}_{+} \times D \\
\Phi(0, x)=u_{0}(x) \text { in } D
\end{array}\right.
$$

where $D$ is a bounded rectangle of $\mathbb{R}^{N}$ and $u_{0}$ and $F$ are given functions. Hereunder we shall use the classical notations for finite difference schemes on regular meshes of points indexed by $i, j$. Starting from $\Phi(0, x)=u_{0}(x)$, then the evolution of $\Phi$ after one time step $\Delta t$ is given by

$$
\Phi_{i j}^{n+1}=\Phi_{i j}^{n}-\Delta t\left(\max \left(F_{i j}, 0\right) \nabla^{+} \Phi+\min \left(F_{i j}, 0\right) \nabla^{-} \Phi\right)
$$

where,

$$
\nabla^{+} \Phi=\left[\max \left(D_{i j}^{-x} \Phi, 0\right)^{2}+\min \left(D_{i j}^{+x} \Phi, 0\right)^{2}+\max \left(D_{i j}^{-y} \Phi, 0\right)^{2}+\min \left(D_{i j}^{+y} \Phi, 0\right)^{2}\right]^{1 / 2}
$$

and

$$
\nabla^{-} \Phi=\left[\max \left(D_{i j}^{+x} \Phi, 0\right)^{2}+\min \left(D_{i j}^{-x} \Phi, 0\right)^{2}+\max \left(D_{i j}^{+y} \Phi, 0\right)^{2}+\min \left(D_{i j}^{-y} \Phi, 0\right)^{2}\right]^{1 / 2}
$$

where,

$$
D_{i j}^{+x} \Phi=\frac{\Phi_{i+1, j}-\Phi_{i, j}}{\Delta x}
$$

for a space step equal to $\Delta x$. The quantities $D_{i j}^{-x} \Phi, D_{i j}^{+y} \Phi$ and $D_{i j}^{-y} \Phi$ are easily deduced. Finally, to define completely our problem, we add the following boundary condition:

$$
\frac{\partial \nabla \Phi(t, x)}{\partial n}=0 \text { on } \partial D
$$

Remark 6. We also considered the boundary condition:

$$
\frac{\partial \Phi(t, x)}{\partial n}=0 \text { on } \partial D
$$

In our situation, it was not possible to detect significant changes between those two types of boundary conditions. 


\subsection{Initialization of the method}

In this kind of minimization problem, the choice of the initial shape can be very important. For example, if we use this level set method for $\lambda_{3}$ starting from an ellipse with eccentricity greater than $\frac{\sqrt{3}}{2}$, the method converges to the union of 3 identical discs which is a local minimum. Therefore, it seems important to start not too far from the global minimum. It is the reason why we have chosen to use a genetic algorithm as a preprocessor. This genetic algorithm is inspired from the ideas developed by Schoenauer see e.g. [9]. We refer to [18] for more details.

\subsection{Handling non simple eigenvalues}

One difficulty we met applying our method was the emergence of multiple eigenvalues. In such situation, eigenvalues are well known to loose their derivability with respect to a boundary variation as well as in the context of the relaxed approach. From a numerical viewpoint, an oscillatory behavior has been observed in the case of multiple eigenvalues.

In order to reduce the oscillatory behavior, we modify our algorithm the following way. Every time we estimated the eigenvalue $\lambda_{m}$ too close from $\lambda_{m-1}$ (i.e. $\left.|\Omega|\left(\lambda_{m}-\lambda_{m-1}\right) \leq 1\right)$, we modified the descent direction by favouring the minimization of $\lambda_{m}+\lambda_{m-1}$ as compared with $\lambda_{m}$. In other words we replaced the vector field $-u_{m}^{2} n$ by $-\left(\frac{u_{m}^{2}+u_{m-1}^{2}}{2}\right) n$.

In Figure 4, the evolution of $\lambda_{4}$ et $\lambda_{3}$ is presented step by step during the minimization of $\lambda_{4}$. A posteriori, it can be observed that all the computed optimal shapes in fact generate at least double eigenvalues. The author believes that this is a general property (but was unable to prove it):

Open problem. Let $\Omega_{k}^{*}$ be the minimizer of the problem $(5)$, then $\lambda_{k-1}\left(\Omega_{k}^{*}\right)=\lambda_{k}\left(\Omega_{k}^{*}\right)$ (let us recall from [11] that we always have $\left.\lambda_{k+1}\left(\Omega_{k}^{*}\right) \neq \lambda_{k}\left(\Omega_{k}^{*}\right)\right)$.

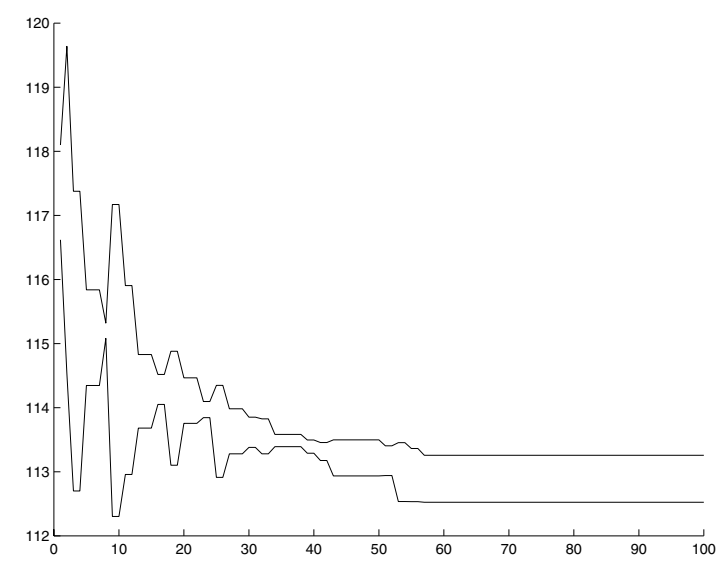

FiguRE 4. Evolution of $\lambda_{3}$ and $\lambda_{4}$.

\subsection{The volume constraint}

The volume of the measure $\mu_{\Phi}$ at the discrete level is by definition the volume of all the elements of the mesh where $\mu_{\Phi}=0$ :

$$
\left|\mu_{\Phi}\right|:=\sum_{T}|T|
$$


where $\mathrm{T}$ is a triangle of the mesh where $\mu_{\Phi}(x) \geq 0$. In order to preserve this volume during iterations, we used the Lagrange multiplier technique reported in [16], which consists in applying the optimization algorithm to the function

$$
L(\Phi, \nu)=\lambda_{k}\left(\mu_{\Phi}\right)+\nu\left|\mu_{\Phi}\right|,
$$

where $\mu_{\Phi}$ is the measure density associated to $\Phi$ by the relation

$$
\mu_{\Phi}(x)=\left\{\begin{array}{l}
0 \text { if } \Phi(x)<0, \\
M \text { otherwise }
\end{array}\right.
$$

where $M$ is a fixed positive constant. According to the derivative computed in Section 4.2, the level set function $\Phi$ satisfies the Hamilton-Jacobi equation

$$
\frac{\partial \Phi}{\partial t}(t, x)-\left(-M u_{k}^{2}(t, x)+\nu\right)|\nabla \Phi(t, x)|=0 \text { in } \mathbb{R}_{+} \times D
$$

where $u_{k}(t,$.$) is the eigenfunction associated to \lambda_{k}\left(\mu_{\Phi(t, .)}\right)$. As suggested by Osher and Santosa in [16], at each iteration we adapted our Lagrange multiplier $\nu$ to preserve the volume constraint.

\subsection{Parameters of the numerical experiments}

In all our experiments on eigenvalues, we used a regular mesh of size $\Delta x=\Delta y=1 / 80$. The system (23) is solved by a classic $P 1$ finite element method. The parameter $M$ (the maximum of the density $\mu$ ) was fixed to the value 800. Usually the algorithm found the minimum in less than 100 iterations (see Fig. 4).

\section{Results AND CONCLUSiON}

The shapes obtained following the combination of the genetic algorithm reported in [9] and the topological optimization by the mixed relaxation/level set method are presented in Figures 6-9 and 10. The quality of the results generated with the two first eigenvalues is particulary satisfying.

For $\lambda_{3}$, it has been conjecture by Szegö (see [24]) that the minimum should be a disc. It is in accordance with our numerical results.

For $\lambda_{4}$, the conjecture states that the minimum is the union of 2 balls whose radii are in the ratio of $\frac{j_{0}}{j_{1}}$ (where $j_{0}$ and $j_{1}$ are the first zeros of the Bessel function $J_{0}$ and $J_{1}$ ). Our method seems to confirm this conjecture.

Szegö raised the question to know whether the minimum was always to be chosen amongst discs or union of discs. The unexpected result obtained for the minimization of $\lambda_{5}$ has to be pointed out. As reported by Wolf and Keller in [24], union of balls do not account for the only optimal shapes. It is noteworthy that this occurs as soon as the 5th eigenvalue (the values reported in Fig. 5 being in fact upper bounds).

On the other hand, let us point out the limit of the process. As already mentioned, the major difficulty we met is the non differentiability of multiple eigenvalues. The strategy proposed in Section 4.5 requires to choose arbitrarily the moment when the vector field has to be modified.

For $\lambda_{7}$, this approach did not allow to identify the shape presented in Figure 5 (see the shape obtained by our method in Fig. 9). Indeed we obtain it using the following theorem of Wolf et Keller: let $k \in \mathbb{N}^{*}$, and for $j=1, \ldots, k$ we define $\lambda_{j}^{*}=\min _{|\Omega|=1} \lambda_{j}(\Omega)=\lambda_{j}\left(\Omega_{j}^{*}\right)$.

Theorem 7 (Wolf-Keller). Let $\Omega_{k}^{*}$ be a non convex open set of $\mathbb{R}^{N}$, which minimizes $\lambda_{k}$ among open sets of volume 1. Then:

and

$$
\left(\lambda_{k}\left(\Omega_{k}^{*}\right)\right)^{N / 2}=\min _{1 \leq j \leq(k-1) / 2}\left(\left(\lambda_{j}^{*}\right)^{N / 2}+\left(\lambda_{k-j}^{*}\right)^{N / 2}\right)
$$

$$
\Omega_{k}^{*}=\left[\left(\frac{\lambda_{i}^{*}}{\lambda_{k}^{*}}\right)^{1 / 2} \Omega_{i}^{*}\right] \cup\left[\left(\frac{\lambda_{k-i}^{*}}{\lambda_{k}^{*}}\right)^{1 / 2} \Omega_{k-i}^{*}\right] .
$$




\begin{tabular}{|c|c|c|}
\hline No & Optimal union of discs & Computed shapes \\
\hline 3 & 46.125 & 46.125 \\
\hline 4 & 64.293 & 64.293 \\
\hline 5 & 82.462 & 78.47 \\
\hline 6 & 92.250 & 88.96 \\
\hline 7 & 110.42 & 107.47 \\
\hline 8 & 127.88 & 119.9 \\
\hline 9 & 138.37 & 133.52 \\
\hline 10 & 154.62 & 143.45 \\
\hline
\end{tabular}

FiguRE 5. Best-known shapes.
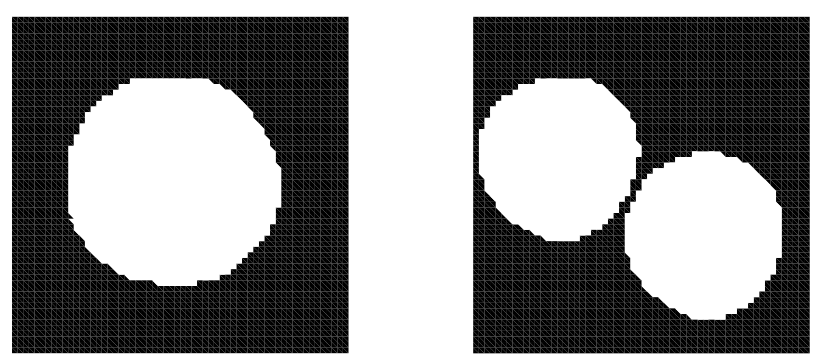

Figure $6 . \lambda_{1}$ (left) and $\lambda_{2}$ (right).
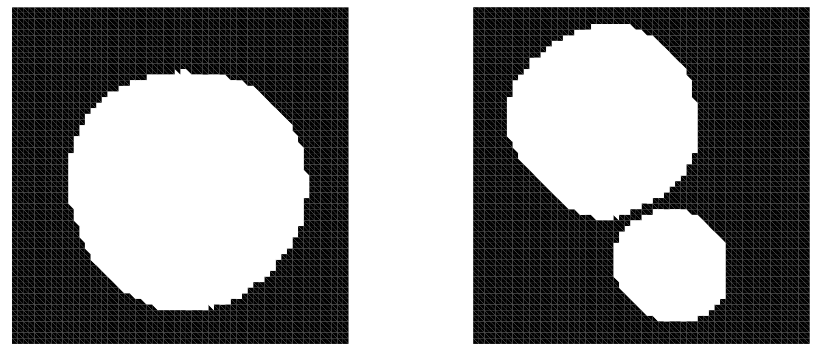

Figure 7. $\lambda_{3}$ (left) and $\lambda_{4}$ (right). 

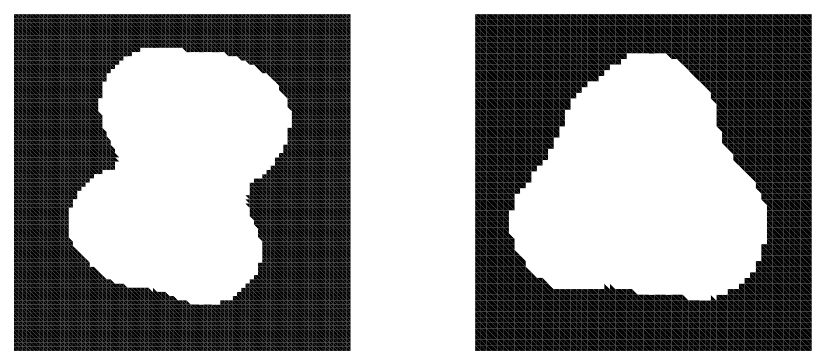

Figure 8. $\lambda_{5}$ (left) and $\lambda_{6}$ (right).
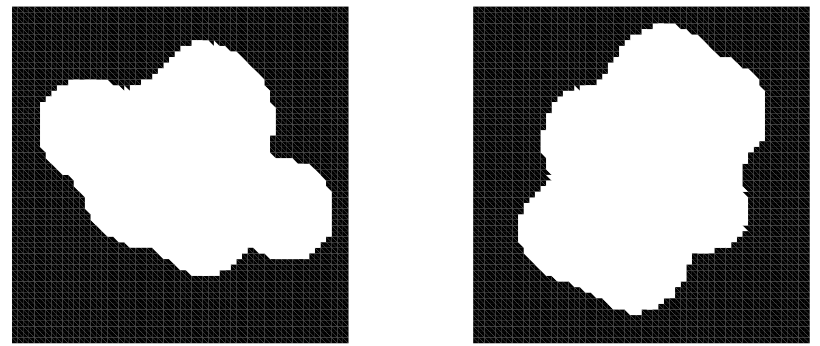

Figure 9. $\lambda_{7}$ (left) and $\lambda_{8}$ (right).
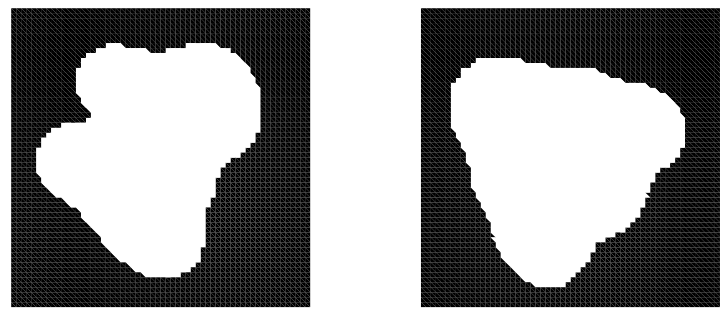

FiguRE 10. $\lambda_{9}$ (left) and $\lambda_{10}$ (right).

So once known the $k$ first optimal domains, the non convex optimal domain minimizing $\lambda_{k+1}$ can be determined. This recursive procedure enabled to identify the shape figured in Figure 5 that has a smaller $\lambda_{7}$ than the one deduced from our algorithm.

Nevertheless, it seems that the combination of the Level Set method of Osher and Sethian with the relaxed approach is quite promising for problems with Dirichlet boundary condition. The author believes that this approach can be applied to a wide variety of shape optimization problems.

\section{REFERENCES}

[1] G. Allaire, Shape optimization by the homogenization method. Springer-Verlag, New York (2001).

[2] G. Allaire, F. Jouve and A.M. Toader, A level-set method for shape optimization. C. R. Acad. Sci. Paris 334 (2002) 1125-1130.

[3] M. Bendsoe, Optimization of structural Topology, Shape and Material. Springer (1995).

[4] M. Bendsoe and C. Mota Soares, Topology optimization of structures. Kluwer Academic Press, Dordrechts (1993).

[5] G. Buttazzo and G. Dal Maso, An Existence Result for a Class of Shape Optimization Problems. Arch. Ration. Mech. Anal. 122 (1993) 183-195.

[6] M.G. Crandall and P.L. Lions, Viscosity Solutions of Hamilton-Jacobi Equations. Trans. Amer. Math. Soc. 277 (1983) 1-43.

[7] G. Faber, Beweis, dass unter allen homogenen Membranen von gleicher Fläche und gleicher Spannung die kreisförmige den tiefsten Grundton gibt. Sitz. Ber. Bayer. Akad. Wiss. (1923) 169-172. 
[8] S. Finzi Vita, Constrained shape optimization for Dirichlets problems: discretization via relaxation. Adv. Math. Sci. Appl. 9 (1999) 581-596.

[9] H. Hamda, F. Jouve, E. Lutton, M. Schoenauer and M. Sebag, Représentations non structurées en optimisation topologique de formes par algorithmes évolutionnaires. Actes du 32e Congrès d'Analyse Numérique, Canum. ESAIM Proc. 8 (2000).

[10] A. Henrot, Minimization problems for eigenvalues of the Laplacian. J. Evol. Eq. 3 (2003) 443-461.

[11] A. Henrot and E. Oudet, Le stade ne minimise pas $\lambda_{2}$ parmi les ouverts convexes du plan. C. R. Acad. Sci. Paris Sér. I Math. 332 (2001) 417-422.

[12] A. Henrot and E. Oudet, Minimizing the second eigenvalue of the Laplace operator with Dirichlet boundary conditions. Arch. Ration. Mech. Anal. 169 (2003) 73-87.

[13] A. Henrot and M. Pierre, Optimisation de forme (in preparation).

[14] E. Krahn, Über eine von Rayleigh formulierte Minimaleigenshaft des Kreises. Math. Ann. 94 (1925) 97-100.

[15] E. Krahn, Über Minimaleigenshaften der Kugel in drei und mehr Dimensionen. Acta Comm. Univ. Dorpat. A9 (1926) 1-44.

[16] S. Osher and F. Santosa, Level set methods for optimization problems involving geometry and constraints: frequencies of a two-density inhomogeneous drum. J. Comput. Phys. 171 (2001) 272-288.

[17] S. Osher and J.A. Sethian, Front propagation with curvature-dependant speed: Algorithms based on Hamilton-Jacobi formulations J. Comput. Phys. 79 (1988) 12-49.

[18] E. Oudet, Quelques résultats en optimisation de forme et stabilisation. Prépublication de l'Institut de recherche mathématique avancée, Strasbourg (2002).

[19] M. Pierre and J.M. Roche, Numerical simulation of tridimensional electromagnetic shaping of liquid metals. Numer. Math. 65 (1993) 203-217.

[20] G. Pólya and G. Szegö, Isoperimetric Inequalities in Mathematical Physics. Ann. Math. Stud. 27 (1952).

[21] J.A. Sethian, Level Set Methods and Fast Marching Methods. Cambridge University Press (1999).

[22] J. Sokolowski and J.P. Zolesio, Introduction to shape optimization: shape sensitivity analysis. Springer, Berlin, Springer Ser. Comput. Math. 10 (1992).

[23] B.A. Troesch, Elliptical membranes with smallest second eigenvalue. Math. Comp. 27 (1973) 767-772.

[24] S.A. Wolf and J.B. Keller, Range of the first two eigenvalues of the laplacian. Proc. Roy. Soc. Lond. A 447 (1994) $397-412$. 